\title{
AN EXPLICIT FAMILY OF EXOTIC CASSON HANDLES
}

\author{
ŽARKO BIŽACA
}

(Communicated by Ronald Stern)

\begin{abstract}
This paper contains a proof that the Casson handle that contains only one, positive, self-intersection on each level, $\mathrm{CH}^{+}$, is exotic in the sense that the attaching circle of this Casson handle is not smoothly slice in its interior. The proof is an easy consequence of L. Rudolph's result (Bull. Amer. Math. Soc. (N.S.) 29 (1993), 51-59) that no iterated positive untwisted doubles of the positive trefoil knot is smoothly slice. An explicit infinite family of Casson handles is constructed by using the non-product h-cobordism from $\check{Z}$. Bižaca ( $A$ handle decomposition of an exotic $\mathbb{R}^{4}$, J. Differential Geom. (to appear)), $\mathrm{CH}_{n}, n \geq 0$, such that $\mathrm{CH}_{0}$ is the above-described $\mathrm{CH}^{+}$and each $\mathrm{CH}_{n+1}$ is obtained by the reimbedding algorithm (Ž. Bižaca, $A$ reimbedding algorithm for Casson handles, Trans. Amer. Math. Soc. 345 (1994), 435-510) in the first six levels of $\mathrm{CH}_{n}$. An argument that no two of those exotic Casson handles are diffeomorphic is outlined, and it mimics the one from S. DeMichelis and M. Freedman (J. Differential Geom. 17 (1982), 357-453).
\end{abstract}

The purpose of this paper is to present explicit examples of exotic Casson handles. For a description of Casson handles the reader is referred to [C], [F], or [K2]. The notion of 'exoticness' is defined below. A way to describe any handle, and so a Casson handle in particular, is to think of it as a pair consisting of a manifold and a selected piece of its boundary, "the attaching area". One of the biggest break-throughs in four-dimensional topology was M. Freedman's proof [F] that every Casson handle is homeomorphic to the standard open 2-handle, $\left(D^{2} \times \mathbb{R}^{2}, S^{1} \times \mathbb{R}^{2}\right)$. Andrew Casson constructed these manifold pairs in an attempt to mimic the proof of the h-cobordism theorem for higher-dimensional manifolds in the setting of four-dimensional manifolds [C]. The piece of the higher-dimensional proof that does not apply to dimension four is the Whitney trick (see [RS, §6]). Casson handles were constructed in places where embedded 2-handles, or "Whitney discs", are needed in order to perform the Whitney trick [C]. Therefore, a consequence of Freedman's result is the topological hcobordism theorem for four-dimensional manifolds [F]; that is, an h-cobordism between two simply-connected closed four-dimensional manifolds, say $M_{0}$ and $M_{1}$, is necessarily homeomorphic to the product cobordism, $M_{0} \times[0,1]$.

If all Casson handles were diffeomorphic to the standard open 2-handle, the

Received by the editors July 28, 1993.

1991 Mathematics Subject Classification. Primary 57M99, 57N13, 57R65.

Key words and phrases. Casson handles, Casson towers, exotic Casson handles, exotic $\mathbb{R}^{4}$, Freedman's reimbedding theorem. 
h-cobordism theorem would be smoothly true [C]; any smooth h-cobordism between simply-connected closed four-manifolds would be diffeomorphic to the product cobordism. By now there are many known counterexamples to the smooth h-cobordism theorem (for some concrete examples see [A] or [Ko]). Obviously any Casson handle that appears as the only Whitney disc in a nonproduct h-cobordism cannot be diffeomorphic to the standard open 2-handle. Also, it cannot happen that the attaching circle of such a Casson handle bounds a smoothly embedded disc inside the Casson handle. It is customary to refer to a Casson handle with this non-embedding property as an exotic Casson handle.

It is still an open conjecture that all Casson handles are exotic. R. Gompf has shown that there are infinitely many diffeomorphism classes of Casson handles (see [G1] and [G2]). However, until recently it was not known for any particular Casson handle whether it is exotic or not. The first concretely described example of an exotic Casson handle was constructed in [B1]. The same Casson handle was used in a construction of a handlebody of an exotic $\mathbb{R}^{4}$ [B2]. (An "exotic $\mathbb{R}^{4}$ " is a smooth manifold homeomorphic but not diffeomorphic to the standard Euclidean four-space, $\mathbb{R}^{4}$.)

Recall that a Casson handle can be constructed as an infinite tower whose levels consist of "kinky handles". Each kinky handle is a manifold pair diffeomorphic to a relative neighborhood of an immersed disc in the four-ball. A kinky handle is completely determined by two numbers: the number of positive and the number of negative self-intersections (or "kinks") on the given immersed disc, or "core". A new level of a tower is formed by attaching kinky handles to cap loops generating the fundamental group of the previous level. For each self-intersection there is a corresponding generator. Also, each of the loops involved is assumed to have a certain canonical framing. The information needed to reconstruct a Casson handle up to diffeomorphism can be encoded in an infinite-based signed tree where each vertex corresponds to a self-intersection, its sign corresponds to the sign of the self-intersection and all edges going up from a given vertex determine the self-intersections of the kinky handle on the next level that was attached over the loop for the given self-intersection. If such a tree for a Casson handle is homeomorphically embedded as a subtree of a tree for another Casson handle, then the Casson handle with the bigger tree can be embedded in the Casson handle with the smaller tree. Such an embedding can be constructed by adding new self-intersection points and new kinky handles to the cores in the simpler Casson handle, as prescribed by the bigger tree. (We will always assume that an embedding between handles preserves attaching areas or that it at least preserves attaching circles and their framings.) Similarly, every Casson handle can be embedded in the standard 2-handle by introducing self-intersections and kinky handles to the core of the standard 2-handle. Of course, for exotic Casson handles there are no embeddings going in the opposite direction. The example of an exotic Casson handle from [B1] corresponds to a huge tree where the number of branchings grows superexponentialy with the level. The following example is as simple as possible.

Theorem A. The Casson handle with only one self-intersection on each level and with only positive self-intersections, $\mathrm{CH}^{+}$, is exotic.

Remark. It is not known at present whether the condition that all self-intersections are positive can be dropped from Theorem A. By taking mirror images 


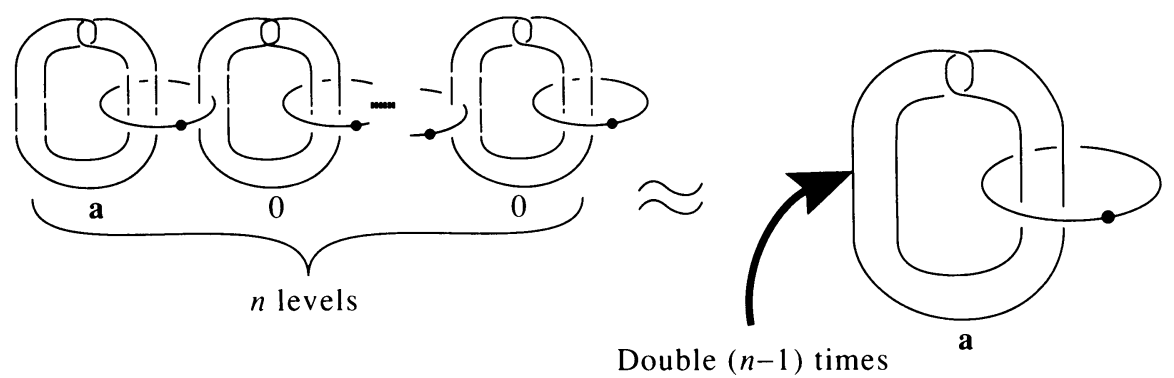

FIGURE 1. Equivalent link calculus pictures of the first $n$ levels of $\mathrm{CH}^{+}$.

it follows that the Casson handle $\mathrm{CH}^{-}$, with one negative self-intersection on each level, is also exotic. If Theorem $A$ is true for Casson handles with one self-intersection of an arbitrary sign on each level, then all Casson handles are exotic. Namely, a Casson handle that embeds in an exotic Casson handle is itself exotic and any Casson handle can be embedded in one whose corresponding tree has only one self-intersection on each level. Notice that by writing the numbers from the unit interval, $[0,1]$, in the binary notation, one can obtain a surjection from the set of Casson handles with only one kink on each level onto the interval $[0,1]$.

Proof of Theorem A. Suppose that $\mathrm{CH}^{+}$is not exotic, i.e., that its attaching circle is smoothly slice. $\mathrm{CH}^{+}$has the limit topology as an infinite union of kinky handles and therefore the slicing disc (being compact) meets only finitely many levels; say it is embedded in the first $n$ levels of $\mathrm{CH}^{+}$, for some positive integer $n$.

Two link calculus pictures (see [K1], [K2], or [F]) of the first $n$ levels of $\mathrm{CH}^{+}$are visible in Figure 1. The standard one on the left ([C], [K2], or [F]) consists of the $n$ Whitehead links, each but the first one formed from a 1-handle (dotted circles) and a 0-framed 2-handle. The component labeled with ' $a$ ' is the attaching circle of the Casson handle $\mathrm{CH}^{+}$for which we assumed that it bounds a smoothly embedded disc inside the handlebody. Contracting $(n-1)$ many complementary pairs of 1- and 2-handles, all but the first and last components of the link disappear and the first component is doubled $(n-1)$ times, that is, the result is an $(n-1)$-fold untwisted positive double of the Whitehead link. The assumption that the attaching circle is slice in the first $n$ levels of $\mathrm{CH}^{+}$ implies that this link is smoothly slice. The manifold described by Figure 2 is obtained by adding a 2-handle that is complementary to the 1-handle from Figure 1, so both can be removed from the picture and the resulting manifold is just a four-ball. The framing of the attached 2-handle is -1 , which results in a right-hand twist being added to the attaching circle. The result is visible as a right-hand trefoil in Figure 3, which is what the picture would be if $n$ was equal to one. In general, since the doubling commutes with the twisting, the picture is $(n-1)$-fold untwisted positive double of the trefoil knot. Since this circle bounded an embedded disc before we added a -1-framed 2-handle, the resulting knot bounds the same embedded disc in the obtained manifold ( $=$ four-ball), or, in other words, $(n-1)$-fold untwisted positive double of the positive trefoil knot is smoothly slice. But this is in contradiction with 


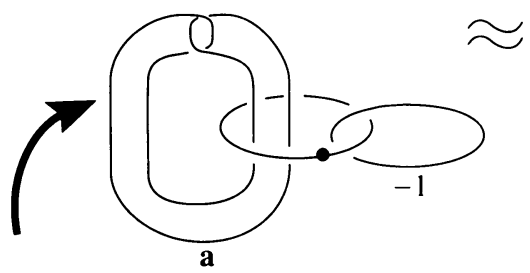

Double $(n-1)$ times

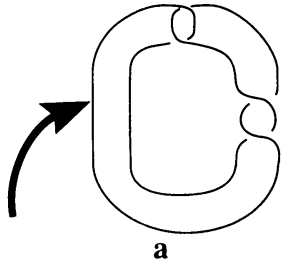

Double $(n-1)$ times

FIGURE 2

FIGURE 3

L. Rudolph's result [R] that no iterated untwisted positive double of the positive trefoil knot is smoothly slice.

We will now show how to use Theorem $A$ to provide an infinite sequence of (potentially explicitly described) exotic Casson handles, of which $\mathrm{CH}^{+}$is the first. In [DF], DeMichelis and Freedman analyzed Kotschick's [Ko] example of a non-product smooth h-cobordism between a pair of non-diffeomorphic one-connected four-dimensional manifolds that are distinguished by having a different value of a gauge-theoretic invariant. They have shown that the two non-diffeomorphic manifolds in the boundary of the cobordism differ by an embedding of an open manifold $R$, an exotic $\mathbb{R}^{4}$. Furthermore, in [DF] it was shown that this Kotschick's invariant can be defined for manifolds with ends periodic in the sense of [T]. Following an argument in [T], one can conclude that there is a compact $K$ in $R$ such that if $R_{n}$ is an open smooth proper submanifold of $R$ with the property that $K \subset R_{n} \subset R$ then pairs $\left(R_{n}, K\right)$ and $(R, K)$ cannot be diffeomorphic by a diffeomorphism that is identity on the compact $K$, and also, there cannot be an embedding $R \hookrightarrow R_{n}$ that is the identity on $K$. Roughly the argument is as follows: a compact $K$ is chosen so that the h-cobordism is smoothly a product over the complement of $K$. If there was such a diffeomorphism or an embedding in the opposite direction between the manifold pairs $\left(R_{n}, K\right)$ and $(R, K)$, then there would exist a self-embedding of $(R, K)$ that is compactly supported and equal to the identity on $K$. As argued in [DF], an iteration of this self-embedding and the removal of the intersection of the images would produce an open manifold with periodic end, embedded in the complement of $K$. This open manifold would have the same value of Kotschick's invariant as the initial, closed manifold [DF]. This construction can be done in both components of the boundary of the cobordism and since the resulting open manifolds are diffeomorphic, then the invariant has to be the same for the closed manifolds. This is not true for the given example, therefore there is no compactly supported self-embedding of $(R, K)$.

Another explicit example of a non-product h-cobordism, due to $\mathrm{S}$. Akbulut [A], was used in [B2] to produce a handlebody for an exotic $\mathbb{R}^{4}$. In this example a different gauge-theoretic invariant is used to distinguish between two manifolds in the boundary of the cobordism, namely a Donaldson polynomial evaluated on fixed second homology classes. However, an argument analogous to the one outlined above can be applied to relate this invariant of closed manifolds with open manifolds with periodic end, obtained by the process above. 
The same non-existence conclusions apply for the similar pairs $\left(R_{n}, K\right)$ and $(R, K)$.

We continue with a rough outline of the construction of an exotic $\mathbb{R}^{4}$ from [B2]. The construction starts with a compact manifold with boundary, denoted by $Y$, whose homotopy is generated by two 1-handles. If those generators are capped by copies of a specific Casson handle the resulting manifold is an exotic $\mathbb{R}^{4}$ [B2, Figures 2 and 4]. The corresponding tree of the Casson handle may be explicitly determined by a sequence of nonnegative integers, but what guarantees that the construction will produce an exotic $\mathbb{R}^{4}$ is that the used Casson handle can be embedded in the first eight levels of $\mathrm{CH}^{+}$. A fixed compact manifold $K$ is constructed by capping the two generators of $Y$ by copies of closure of the first three levels of $\mathrm{CH}^{+}$. This guarantees that the interior of $K$ contains an exotic $\mathbb{R}^{4}$ and that the cobordism from [A] is a product over the complement of $K$. Then, the reimbedding algorithm from [B1] produces a Casson handle embedded in the first six levels of $\mathrm{CH}^{+}$. Call the resulting Casson handle $C H_{1}$. Two copies of $C H_{1}$ are added to $K \cup(\partial K \times$ $[0, \infty)$ ) by identifying the open kinky handle (with only one, positive, selfintersection) from the third level of $\mathrm{CH}^{+}$in $\mathrm{K}$ with the first level of $\mathrm{CH}_{1}$, which is possible since the reimbedding algorithm always preserves the first level. The resulting open smooth submanifold is denoted by $R_{1}$ and contains $K$. Because $R_{1}$ can be embedded in $K \cup 2$ (first eight levels of $C H^{+}$), $R_{1}$ is an exotic $\mathbb{R}^{4}$. The process can be repeated to construct $R_{2}, R_{3}, \ldots$ iteratively: $R_{n+1}$ is constructed by finding a Casson handle $C H_{n+1}$ with the property that it can be embedded with compact support in $\mathrm{CH}_{n}$; for example, the reimbedding algorithm can be used with the first six levels of $C H_{n}$ as the input. Then as before, $R_{n+1}=K \cup(\partial K \times[0, \infty)) \cup_{2} C H_{n+1}$ and $K \subset R_{n+1} \subset R_{n}$. It is clear from the argument above that there cannot be a diffeomorphism fixed on $K$ between different pairs $\left(R_{n}, K\right)$ and $\left(R_{m}, K\right)$. Also all embeddings can go only in the one direction; if $m>n$, then there is no embedding $R_{m} \hookrightarrow R_{n}$ that is the identity on $K$. Any diffeomorphism between $C H_{n}$ and $C H_{m}$ or an embedding of $\mathrm{CH}_{n}$ in $\mathrm{CH}_{m}$, for $n<m$, can be extended over $R_{n}$ and $R_{m}$ by taking it to be identity over $K$. Therefore we have proved the following corollary.

Corollary B (Compare with [G2, Proposition 4.1]). Let $\left\{C H_{n}\right\}_{n=0}^{\infty}$ be a sequence of Casson handles such that:

(i) $\mathrm{CH}_{0}=\mathrm{CH}^{+}$;

(ii) $\mathrm{CH}_{1}$ embeds in the first six levels of $\mathrm{CH}_{0}$; and

(iii) $C H_{n+1}$ embeds in a compact subset of $\mathrm{CH}_{n}$.

Then, for $k<n, C H_{n}$ does not embed in $\mathrm{CH}_{k}$ and in particular, no $\mathrm{CH}_{n}$ and $\mathrm{CH}_{k}$ are diffeomorphic for different $k$ and $n$.

Remark. The existence of a Casson handle that might play the role $\mathrm{CH}_{0}$ in Corollary B was proven by Gompf [G2] and the novelty here is an explicit description of such an $\mathrm{CH}_{0}$. Note that by using the reimbedding algorithm from [B1] one can produce in Corollary B a sequence $\left\{C H_{n}\right\}_{n=0}^{\infty}$ of explicitly described Casson handles. 


\section{REFERENCES}

[A] S. Akbulut, $A$ fake contractible 4-manifold, J. Differential Geom. 33 (1991), 335-356.

[B1] Ž. Bižaca, A reimbedding algorithm for Casson handles, Trans. Amer. Math. Soc. 345 (1994), 435-510.

[B2] _ $A$ handle decomposition of an exotic $\mathbb{R}^{4}$, J. Differential Geom. 39 (1994), 491-508.

[C] A. Casson, Three lectures on new infinite construction in 4-dimensional manifolds (notes prepared by L. Guillou), A la Recherche de la Topologie Perdue (L. Guillou and A. Marin, eds.), Progr. Math., vol. 62, Birkhäuser, Boston, 1986, pp. 201-244.

[DF] S. DeMichelis and M. Freedman, Uncountably many exotic $R^{4}$ 's in standard 4-space, J. Differential Geom. 35 (1992), 219-254.

[F] M. Freedman, The topology of 4-dimensional manifolds, J. Differential Geom. 17 (1982), 357-453.

[G1] R. Gompf, Infinite families of Casson handles and topological disks, Topology 23 (1984), 395-400.

[G2] __ Periodic knots and knot concordance, Topology Appl. 32 (1989), 141-148.

[K1] R. Kirby, A calculus for framed links in $S^{3}$, Invent. Math. 45 (1978), 35-56.

[K2] _ The topology of 4-manifolds, Lecture Notes in Math., vol. 1374, Springer-Verlag, New York, 1989.

[Ko] D. Kotschick, On manifolds homeomorphic to $\mathbb{C} P^{2} \sharp 8 \overline{\mathbb{C P}}^{2}$, Invent. Math. 95 (1989), 591-600.

[RS] C. Rourke and B. Sanderson, Introduction to piecewise-linear topology, Ergebnisse der Math., Springer-Verlag, New York, 1972.

[R] L. Rudolph, Quasipositivity as an obstruction to sliceness, Bull. Amer. Math. Soc. (N.S.) 29 (1993), 51-59.

[T] C. Taubes, Gauge theory on asymptotically periodic 4-manifolds, J. Differential Geom. 25 (1987), 363-430.

Department of Mathematics and Computer Science, Clark University, Worcester, MASSACHUSETTS 01610

Current address: Department of Mathematics, University of Texas, Austin, Texas 78712

E-mail address: bizaca@math.utexas .edu 